\title{
Le disfunzioni metacognitive nei disturbi di personalità: Una review delle ricerche del III Centro di Psicoterapia Cognitiva
}

\author{
Raffaele Popolo, ${ }^{1}$ Antonio Semerari, ${ }^{1}$ Antonino Carcione, ${ }^{1}$ \\ Donatella Fiore, ${ }^{1}$ Giuseppe Nicolò, ${ }^{1}$ Laura Conti, ${ }^{1}$ Roberto Pedone, ${ }^{1}$ \\ Michele Procacci, ${ }^{1}$ Stefania d'Angerio ${ }^{1}$ e Giancarlo Dimaggio ${ }^{1}$
}

\section{Sommario}

Dagli anni '90, il III Centro di Psicoterapia Cognitiva di Roma si è impegnato nell'attività clinica e di ricerca sul trattamento di pazienti gravi e difficili da trattare. In questo lavoro verranno analizzati alcuni tra i più importanti lavori pubblicati dal Gruppo in questo ambito. Si tratta di ricerche sul processo terapeutico condotte a partire dall'osservazione clinica secondo cui la presenza di specifici malfunzionamenti metacognitivi ostacolerebbe la costruzione della rappresentazione degli stati mentali propri e altrui; la regolazione della relazione terapeutica potrebbe migliorare tali malfunzionamenti rendendo così possibili gli interventi sugli aspetti sintomatici del paziente. Sono state portate, quindi, prove sufficienti a sostenere che la metacognizione sia una grandezza composta da sottofunzioni distinte semi-indipendenti. Altri studi del Gruppo hanno mostrato poi come la realtà clinica del paziente grave sia influenzata in modo diverso dalla presenza di specifici malfunzionamenti metacognitivi.

\section{Parole chiave}

Metacognizione, disturbi di personalità, ricerche di processo

${ }_{1}^{1}$ Terzo Centro di Psicoterapia Cognitiva, Roma - Scuola di Psicoterapia Cognitiva (SPC)

Corrispondenza: Raffaele Popolo E-mail: popolo.r@libero.it

Tel.: 0644233878 - Cell: 3398627604 


\section{Introduzione}

A metà degli anni '90 un gruppo di colleghi si trovava a discutere con passione di casi clinici nell'aula del proprio training formativo in psicoterapia cognitiva; si parlava non di terapie di successo, ma di pazienti difficili che a differenza di altri sembravano non essere in grado di ragionare in termini di stati mentali: pazienti complessi che rendevano ancor più difficile la relazione terapeutica. Tale confronto è stato poi spostato dall'aula di lezione al campo della ricerca: nasce così a Roma il Terzo Centro di Psicoterapia Cognitiva. Un legame nato intorno allo stimolo portato loro dai pazienti gravi, pazienti che non rispondevano prontamente ai protocolli cognitivi standard, e che lasciavano per questo nei terapeuti molte domande (oltre che molti problemi!). Le difficoltà incontrate nel trattamento di questi pazienti hanno spinto gli autori ad analizzare i complessi fenomeni che caratterizzano la relazione terapeutica. Questi pazienti avevano difficoltà a riconoscere i propri pensieri ed emozioni, ad attribuire senso alle parole del terapeuta e quindi a reagire in modo adeguato ai suoi interventi. Al tempo stesso, il terapeuta tendeva a volte ad agire in modo disfunzionale con questi pazienti, mosso da emozioni che incidono negativamente sulla qualità della relazione.

Gli autori hanno quindi iniziato a registrare e trascrivere le sedute di psicoterapia e a effettuare la supervisione direttamente sul materiale clinico raccolto. Dallo studio dei trascritti raccolti è emerso che spesso i pazienti con disturbi di personalità presentano difficoltà costanti in specifiche funzioni metacognitive. Per funzioni metacognitive si intendono tutte quelle abilità che consentono alle persone di attribuire e riconoscere stati mentali in sé e negli altri a partire da espressioni facciali, stati somatici, comportamenti e azioni; di riflettere e ragionare sugli stati mentali e di utilizzare le informazioni sugli stati mentali per decidere, risolvere problemi o conflitti psicologici e interpersonali e padroneggiare la sofferenza soggettiva (Carcione, Semerari, Nicolò, 
Pedone, Popolo, Conti, Fiore, Procacci, \& Dimaggio, 2010). Tali osservazioni hanno spinto gli autori a cercare un modo per affrontare questo problema, seguendo un percorso in parte già tracciato da Fonagy (1991) o Frith (1992); essi ritenevano infatti che buone capacità metacognitive fossero necessarie per una terapia efficace, e che in caso di una loro carenza andassero promosse affinché la terapia potesse aver successo (Semerari, 1999).

\section{La Scala di Valutazione della Metacognizione - SVaM}

Il lavoro del III Centro si è quindi concentrato a creare e validare uno strumento di ricerca che fosse in grado di misurare le funzioni metacognitive nel corso di una psicoterapia. Alla base dello strumento vi erano due ipotesi formulate sulla base della discussione clinica di casi singoli. La prima, a differenza di quanto sostenuto da altri autori (Ryle \& Kerr, 2002; Fonagy, Gergely, Jurist, \& Target, 2002; Sperber, 2000), assumeva che la metacognizione fosse un sistema composto da diversi sotto-sistemi in interazione tra loro e che il malfunzionamento metacognitivo non fosse quindi omogeneo da paziente a paziente (Semerari, Carcione, Dimaggio, Falcone, Nicolò, Procacci, \& Alleva, 2003; Semerari, Carcione, Dimaggio, Nicolò, \& Procacci, 2007; Dimaggio \& Lysaker, 2010). Alcuni pazienti avevano gravi difficoltà nel tradurre lo stato somatico in linguaggio affettivo ("ho come una morsa allo stomaco costante") e a spiegare le cause e le motivazioni delle proprie azioni ("oggi sono stato di pessimo umore, sarà la luna ostile"), altri invece erano incapaci di distinguere una fantasia disturbante (" $i$ colleghi mi perseguitano") dalla realtà delle cose. La seconda ipotesi era che i diversi tipi di danno metacognitivo influenzassero in modo diverso il quadro clinico e la relazione terapeutica. Ad esempio, pazienti con difficoltà nel riconoscimento delle emozioni e dei pensieri tendono al ritiro dalle relazioni e, nelle loro terapie, vi sono frequenti momenti di distacco nella relazione terapeutica; altri pazienti che presentano 
difficoltà a riflettere su pensieri ed emozioni in modo integrato tendono invece a sviluppare relazioni caotiche e coinvolgenti tanto nella vita quanto nella relazione col terapeuta. In sintesi, l'idea era che la metacognizione fosse costituita da un insieme di processi ad un tempo collegati concettualmente, ma semi-indipendenti, ovvero che potessero essere funzionanti o danneggiati in modo relativamente autonomo l'uno dall'altro.

In precedenza erano stati sviluppati diversi strumenti atti a valutare la metacognizione, differenti tra loro per proprietà psicometriche e oggetto d'indagine. Tra questi, ad esempio, prove di laboratorio che valutare valutavano vari aspetti della Teoria della Mente, dalla capacità di cogliere che gli altri possono avere convinzioni differenti dalle proprie alla comprensione delle metafore o dell'ironia (vedi ad esempio Perner $\&$ Wimmer, 1985). Altri strumenti, invece, erano questionari autosomministrati che consentivano un rapido studio di un gran numero di pazienti: tra questi, ad esempio, il Meta-Cognition Questionnaire (Cartwright-Hatton \& Wells, 1997) che indagava le convinzioni metacognitive dei pazienti; oppure la TAS-20 utilizzata per valutare la capacità del soggetto di descrivere le proprie emozioni (Bagby, Parker, \& Taylor, 1994a, 1994b). Questi strumenti presentavano però, a nostro avviso, alcuni limiti nell'applicazione alla ricerca in psicoterapia. Le valutazioni venivano fatte su prove effettuate in un contesto rassicurante come quello di uno studio, diverso da quello reale dove invece la sintomatologia si esprime; i risultati ottenuti potrebbero allora non descrivere fedelmente quel funzionamento che il paziente avrebbe nelle situazioni problematiche, sottoposto alla pressione di emozioni negative, visto che le abilità metarappresentative risentono del clima interpersonale. In particolare, i questionari non fornivano informazioni circa il funzionamento del paziente. Per poter rispondere è necessario avere una comprensione dello scopo specifico della domanda, un accesso chiaro alle proprie rappresentazioni, l'abilità 
di costruire una rappresentazione nuova che integri in modo fluido $\mathrm{i}$ propri contenuti con quelli della domanda: abilità strettamente metacognitive, non necessariamente funzionanti in un paziente. Gli autori hanno allora elaborato la Scala di Valutazione della Metacognizione (SVaM; Carcione, Falcone, Magnolfi, \& Manaresi, 1997; Semerari et al., 2003), disegnata per valutare gli aspetti metacognitivi a partire dalla verbalizzazione dei pazienti nel corso delle sedute di psicoterapia. Gli scopi erano quelli di identificare le disfunzioni nella metacognizione presenti nei pazienti, valutare se esse differissero da paziente a paziente e se i trattamenti di successo fossero collegati a un loro miglioramento. Si voleva poi investigare se alcuni aspetti della disfunzione fossero più facilmente influenzati dal trattamento (in alcune categorie di pazienti), a differenza di altri più resistenti, che assumono vera e propria caratteristica di deficit.

La SVaM è divisa in tre sezioni che si riferiscono rispettivamente alle abilità di: Autoriflessività, che comprende le operazioni di conoscenza dei propri stati mentali; Comprensione della mente altrui, che riguarda le operazioni di conoscenza degli stati mentali altrui (tra queste si segnala in particolare il Decentramento, ovvero la capacità di descrivere gli stati mentali altrui a prescindere dal proprio punto di vista o coinvolgimento nella relazione); Mastery, che riguarda le strategie con cui il soggetto tenta di fronteggiare la sofferenza psicologica e i problemi interpersonali. Ogni sezione, a sua volta, comprende poi varie sottofunzioni. La SVaM può essere applicata a trascritti di seduta o a interviste che stimolano la rievocazione di brani o racconti; vengono siglati successi e fallimenti del paziente in relazione a ciascuna funzione. La rilevazione è organizzata per ciascuna seduta e i dati rilevati sono le frequenze degli eventi manifestatisi. Attualmente negli studi in corso la modalità di attribuzione dei punteggi è cambiata e si valutano brani più estesi di discorso, ad esempio un terzo di seduta o un'intera Adult Attachment Interview. Ciascuna sottofunzione viene 
siglata come ingaggiata o meno all'interno del brano; nel caso che sia utilizzata, viene valutata con una scala Likert da 1 a 5 a seconda della articolazione con cui la persona esprime la sottofunzione in questione (Carcione et al., 2010).

La SVaM, applicata esclusivamente a ricerche single case e quindi con i limiti propri di tale metodologia, ci ha permesso di identificare precisi indicatori di processo e di esito in psicoterapia, di delineare specifici profili metacognitivi caratteristici di determinati disturbi di personalità. Tale strumento si è mostrato efficace nella ricerca in psicoterapia in quanto ha il vantaggio di basarsi sulla trascrizione di sedute audio-registrate rendendo il materiale stesso verificabile. È stato possibile poi trattare $\mathrm{i}$ dati in modo statistico permettendo cosi di confrontare i dati emersi con quelli riportati dalla letteratura, per tradurli poi in pratica clinica.

\section{La SVaM: gli studi principali}

Nella prima fase della ricerca gli autori hanno cercato di portare un contributo alla comprensione dell'architettura del funzionamento metacognitivo, questo a partire dall'analisi di sedute effettuate con la SVaM (Semerari et al., 2003). La conferma dell'esistenza di sottofunzioni parzialmente indipendenti capaci di influenza reciproca, avrebbe condizionato $i$ modelli di trattamento in quanto una psicoterapia, per aver successo, avrebbe dovuto puntare ad affrontare o le sottofunzioni più compromesse o quelle che generavano effetti negativi a palla di neve sugli altri elementi del sistema metacognitivo. In un primo lavoro (Semerari et al., 2003) è stato analizzato un campione di circa 100 sedute di due pazienti aventi una diversa diagnosi di disturbo di personalità (un caso con disturbo borderline e l'altro con disturbo narcisistico). La valutazione, effettuata con la SVaM, ha portato dati a favore dell'ipotesi modulare: in entrambi i pazienti ciascuna funzione presentava un grado differente di compromissione e 
un andamento nel tempo diverso. I due pazienti avevano disfunzioni nettamente differenti: nel paziente borderline, si osservava un malfunzionamento significativo dell'autoriflessività nella differenziazione (capacità di distinguere tra diversi tipi di rappresentazioni e tra rappresentazione e realtà) e nell'integrazione (abilità di riflettere sugli stati mentali e sui contenuti dell'esperienza soggettiva in modo da costruire una visione integrata di sé); il paziente narcisista mostrava invece, sempre nell'autoriflessività, difficoltà nell'identificazione (capacità di descrivere i propri pensieri ed emozioni) e nella relazione tra variabili (capacità di descrivere le cause che generano un'emozione, un pensiero, un comportamento). Tale osservazione ha portato a effettuare altri studi single case, in particolare nei disturbi di personalità, per verificare l'ipotesi dell'esistenza di un profilo prototipico metacognitivo per ciascun disturbo; studi questi che hanno permesso di verificare contestualmente la validità degli strumenti di valutazione elaborati dal Gruppo di ricerca, promovendo una loro costante revisione sulla base dei dati progressivamente raccolti.

Inizialmente sono stati studiati i disturbi di personalità presentati dai pazienti che afferivano direttamente al Centro. Uno studio single case condotto su di un paziente affetto da Disturbo Paranoide di Personalità ha permesso di approfondire il ruolo svolto dal malfunzionamento metacognitivo nella genesi e nel mantenimento dei diversi disturbi (Nicolò, Centenero, Nobile, \& Porcari, 2002). Il paziente analizzato presentava un profilo metacognitivo caratterizzato da malfunzionamenti nel decentramento e nella differenziazione. È stata poi utilizzata la Griglia degli Stati Problematici (Semerari et al., 2003b), strumento che consente l'individuazione dei singoli componenti (temi di pensiero, emozioni, sensazioni somatiche) degli stati mentali problematici di un paziente a partire dalle sue narrazioni; questa ha permesso di identificare gli elementi di contenuto caratteristici del paziente paranoide analizzato: i temi prevalenti erano il senso di 
Costrizione e la Minaccia, mentre le emozioni più rilevanti in questo paziente erano quelle di Paura-Ansia, più della Rabbia che, pur essendo presente, non era pervasiva e intensissima. L'andamento delle funzioni metacognitive era correlato a quello degli stati mentali problematici; il miglioramento delle funzioni metacognitive era poi correlato al successo del trattamento. A un follow up dopo 5 anni dalla fine della terapia il paziente non mostrava più un disturbo paranoideo di personalità pienamente espresso: il miglioramento delle funzioni metacognitive, soprattutto di decentramento e di differenziazione, ha permesso al paziente di gestire più efficacemente quel tratto di sospettosità, diffidenza, e di chiusura sociale che ha continuato a esperire.

Questo e altri studi single case effettuati nella fase iniziale della ricerca hanno prodotto numerosi dati clinicamente rilevanti che andavano a sostenere un'ipotesi originale di architettura della metacognizione; tale ipotesi aveva il valore di partire dall'esperienza clinica e per questo era facilmente condivisibile con altri colleghi. A partire da ciò gli autori si sono interrogati circa l'esistenza di malfunzionamenti metacognitivi nucleari comuni a diversi pazienti con lo stesso disturbo, e che caratterizzano quindi il disturbo rispetto ad altri, pur nel rispetto dell'idea che ciascun individuo presenta un proprio funzionamento che può mostrare numerose differenze rispetto ad altri pazienti con la stessa diagnosi. Semerari e collaboratori (2005) hanno allora analizzato le sedute del primo anno di terapia di 4 pazienti con disturbo borderline di personalità utilizzando la SVaM. I risultati ottenuti, in linea con la letteratura (Fonagy, 1991; Dimaggio \& Semerari, 2001, 2004; Liotti, 2002; Perris \& Skagerlind, 1998; Ryle, 1997) confermavano la presenza di un malfunzionamento metacognitivo in questi pazienti; non si trattava però di una compromissione globale dell'abilità, ma solo di specifiche sottofunzioni. I 4 pazienti, infatti, mostravano una difficoltà a integrare aspetti della propria esperienza, così come avevano già osservato alcuni autori (Kernberg, 1975; Clarkin, 
Yeomans \& Kernberg, 1999; Ryle, 1997; Liotti, 2002; Dimaggio \& Semerari, 2004); tendevano poi a non essere in grado a differenziare tra fantasia e realtà e a considerare le proprie rappresentazioni come un riflesso fedele della realtà, coerentemente con l'ipotesi di Fonagy \& Target (1996) secondo cui i pazienti borderline hanno la tendenza a perdere la loro abilità di distinguere tra fantasia e realtà. Da questa ricerca sembrava emergere un profilo prototipico del disturbo borderline caratterizzato da un malfunzionamento delle sottofunzioni autoriflessive dell'integrazione e della differenziazione. Ciascun paziente presentava poi propri malfunzionamenti metacognitivi che si andavano ad aggiungere a quelli descritti precedentemente (come ad esempio nel monitoraggio in uno dei casi) e che non rappresentavano quindi tratti nucleari del disturbo. Il comportamento di ciascuna funzione non si manteneva poi sempre costante nel tempo. Se consideriamo, ad esempio, la funzione integrazione si è visto che questa, ad eccezione di un caso, poteva passare da momenti in cui era molto compromessa ad altri in cui funzionava meglio; la valutazione qualitativa del trascritto delle sedute mostrava come l'andamento oscillatorio del malfunzionamento era legato a fattori esterni, eventi di vita contestuali e a variabili relazionali e intersoggettive.

Questo studio sostiene l'idea di un malfunzionamento metacognitivo che interessa sottofunzioni specifiche e che si mantiene a lungo nel tempo; da qui l'ipotesi che nei disturbi "meno gravi” la presenza di un malfunzionamento metacognitivo non sia un fattore nucleare come nei disturbi di personalità, ma si possa presentare in determinati contesti problematici contribuendo all'espressione sintomatologica del disturbo.

Diversi autori sostengono infatti che la metacognizione sia solo marginalmente compromessa in pazienti con disturbi "nevrotici", come ad esempio nella depressione (Honkalampi, Hintikka, Antikainen, Lehtonen, \& Viinamäki, 2001; Inoue, Tonooka, Yamada, \& Kanba, 2004; Inoue, Yamada, \& Kanba, 2006); questi pazienti presenterebbero 
invece una maggiore compromissione della capacità di padroneggiamento dei problemi psicologici (Carcione, Dimaggio, Fiore, Nicolò, Procacci, Semerari, \& Pedone, 2008). Al fine di verificare tale ipotesi, gli autori hanno valutato la metacognizione in una paziente con diagnosi di depressione usando anche in questo caso la SVaM (Carcione et al., 2008). La paziente ha mostrato solo minimi fallimenti metacognitivi, rilevati in particolare nella fase iniziale della terapia; aveva invece difficoltà a padroneggiare i propri stati problematici, dato sostenuto dai fallimenti nella mastery osservati. Queste difficoltà, a differenza di quanto visto nei pazienti con disturbo di personalità, miglioravano rapidamente nel corso del trattamento parallelamente al miglioramento del quadro depressivo.

Riassumendo, le funzioni metacognitive possono essere danneggiate indipendentemente l'una dall'altra e con un grado di compromissione differente nei diversi disturbi. Possono essere funzionanti ma fallire a seguito dell'attivazione emotiva o nell'ambito di situazioni relazionali problematiche, come accade nei disturbi d'ansia o dell'umore; oppure il danneggiamento di queste funzioni può essere più strutturale e costituire un tratto specifico della persona stessa, comportando una compromissione del funzionamento psicosociale, come si osserva nei disturbi di personalità. Diversi pazienti con disturbo di personalità presentavano ciascuno un proprio profilo metacognitivo (Dimaggio \& Semerari, 2003; Dimaggio, Semerari, Carcione, Nicolò, \& Procacci, 2007; Dimaggio, Carcione, Petrilli, Procacci, Semerari, \& Nicolò, 2005; Semerari et al., 2003); si è cercato allora di porre a confronto l'andamento delle singole funzioni nei diversi disturbi di personalità, al fine di valutarne il possibile andamento differente.

In un successivo lavoro Dimaggio et al. (2007) hanno valutato il funzionamento metacognitivo di 4 pazienti, due con disturbo narcisistico e due con disturbo evitante di personalità. Tre dei quattro pazienti valutati (i due pazienti narcisisti e il paziente evitante che 
presentava tratti schizoidi) mostravano difficoltà nel definire i propri stati interni così come a metterli in relazione a fattori psicologici o del contesto; era presente solo una modesta compromissione dell'integrazione, funzione che migliorava in tutti alla fine del primo anno di terapia. L'andamento delle sottofunzioni relative all'autoriflessività era quindi differente rispetto, ad esempio, a quello rilevato nel campione di quattro pazienti borderline studiati da Semerari et al. (2005); nessuno di questi ultimi pazienti presentava infatti un malfunzionamento dell'identificazione o della sottofunzione relazione tra variabili. I pazienti con disturbo borderline mostravano invece una grave compromissione nella differenziazione e nell'integrazione; in particolare, quest'ultima appariva pervasiva e duratura, tanto che solo un paziente borderline mostrava un miglioramento della funzione alla fine del primo anno di terapia. Il confronto tra profili metacognitivi di differenti disturbi ha evidenziato, in questo caso, la presenza di specifici malfunzionamenti nell'autoriflessività, che sembrano essere quindi tratti nucleari dei diversi disturbi (Semerari, 1999). Questa rilevazione, non solo portava dati a conferma di un'ipotesi modulare della metacognizione, ma sottolineava l'opportunità di verificare l'andamento delle altre funzioni metacognitive nei diversi disturbi di personalità; tra queste, in particolare il decentramento, funzione che clinicamente appare compromessa in numerosi pazienti.

Un numero crescente di dati sostiene l'idea che la difficoltà a comprendere gli stati mentali altrui sia una dimensione comune che sottende i disturbi di personalità; la difficoltà a comprendere gli altri, a "mettersi nei loro panni", è stata descritta nei disturbi di personalità (Krueger, Skodol, Livesley, Shrout, \& Huang, 2007; Dimaggio et al., 2007) così come nei loro familiari (Guttman \& Laporte, 2002). Dimaggio, Carcione, Nicolò, Conti, Fiore, Pedone, Popolo, Procacci, \& Semerari (2009) hanno allora analizzato le prime 16 sedute di un campione di 14 pazienti con disturbo di personalità per valutare la loro capacità a 
comprendere la mente degli altri e in particolare l'andamento del decentramento. Il campione era costituito da pazienti con disturbo borderline, narcisistico, paranoide, evitante, ossessivo compulsivo e dipendente di personalità. Nel complesso, dai dati è emerso che i pazienti analizzati avevano tutti difficoltà ad assumere la prospettiva dell'altro nel corso delle relazioni, ad aver chiaro che gli altri agiscono mossi da motivazioni proprie indipendentemente dal rapporto che possono avere in quel momento con loro. Fallivano quindi nel decentrare mentre, al contrario, erano capaci a cogliere i pensieri e le emozioni altrui, ovvero a comprendere la mente degli altri, funzione questa solo occasionalmente danneggiata. I pazienti con disturbo di personalità apparivano allora generalmente egocentrici; il malfunzionamento del decentramento, tranne in pochi casi, si manteneva nell'arco di tempo osservato. La compromissione del decentramento sembra essere un tratto che accomuna i diversi disturbi di personalità studiati, un danneggiamento stabile e apparentemente grave che necessita di tempo per essere modificato. La tendenza a essere egocentrici in maniera patologica rende problematiche le dinamiche relazionali; possiamo ipotizzare che un buon accesso ai propri stati interni e una corretta comprensione della mente altrui metta a disposizione della persona quelle informazioni necessarie a padroneggiare al meglio le situazioni di difficoltà e sofferenza. I fallimenti descritti nell'autoriflessività così come nel decentramento, porterebbero allora $\mathrm{i}$ pazienti a ricorrere a strategie disfunzionali di gestione degli stati problematici.

Al fine di verificare questa ipotesi clinica, Carcione e collaboratori (2010) hanno valutato l'abilità di padroneggiare gli stati mentali problematici e l'andamento di tale capacità nel corso della terapia in 14 pazienti con disturbo di personalità (borderline, narcisistico, evitante, dipendente, ossessivo-compulsivo, paranoide). Il campione di pazienti valutato ha mostrato complessivamente una difficoltà significativa a 
ricorrere a strategie di padroneggiamento che richiedevano capacità sofisticate di comprensione degli stati mentali e usarli a fini di problem solving, quali regolare l'attenzione cosciente o utilizzare la conoscenza degli stati mentali propri e altrui per regolare problemi interpersonali; tale difficoltà non diminuiva rapidamente nell'arco di tempo analizzato (le prime 16 sedute). L'utilizzo della scala della mastery della SVaM, che distingue le diverse strategie in categorie separate secondo una complessità crescente delle operazioni metacognitive implicate, ha permesso poi di verificare come il malfunzionamento della mastery non fosse omogeneo tra i pazienti (Semerari et al., 2007), ma che alcuni mostravano maggiori difficoltà ad affrontare i problemi rispetto ad altri, utilizzando ciascuno strategie di padroneggiamento differenti. Da un punto di vista qualitativo i pazienti si distinguevano sia nel tipo di strategia che tendevano ad usare più frequentemente, sia nel tipo di strategia che usavano più efficacemente. Complessivamente, le strategie utilizzate con maggior efficacia all'inizio del trattamento erano quelle che richiedevano una minima conoscenza degli stati mentali; queste consistevano in interventi concreti, essenzialmente di tipo comportamentale, che venivano attuati sulla situazione in corso, come evitare consapevolmente una situazione temuta. Nelle fasi precoci di terapia si poteva osservare un lieve miglioramento della mastery, ma in gran parte dei casi analizzati il problema persisteva; questo dato lascia supporre che la terapia dei disturbi di personalità richieda un lungo periodo per produrre cambiamenti duraturi nelle abilità di mastery: per poter utilizzare strategie di mastery di livello superiore, il paziente dovrebbe infatti avere un buon accesso ai propri stati interni così come una buona capacità di leggere la mente altrui.

Riassumendo, la SVaM si è dimostrata uno strumento efficace in studi di processo per valutare in modo intensivo il funzionamento metacognitivo dei pazienti in trattamento psicoterapeutico all'interno di protocolli di ricerca single case. Ha permesso di raccogliere dati a 
sostegno di un'ipotesi modulare del funzionamento metacognitivo e di seguire il differente andamento delle singole funzioni nei diversi pazienti con disturbi di personalità. Il decentramento e le strategie di mastery sembrano essere compromesse in tutti questi pazienti, anche se con un grado di gravità del danneggiamento differente in ciascuno. La compromissione di specifiche sottofunzioni dell'autoriflessività sembra invece caratterizzare, e quindi distinguere tra loro, i diversi disturbi di personalità.

\section{L'Intervista per la Valutazione della Metacognizione - IVaM}

Per poter generalizzare tali osservazioni era necessario estendere i progetti di ricerca a campioni di pazienti più numerosi rispetto a quelli analizzati finora; per questo motivo si è resa necessaria la costruzione di uno strumento di valutazione del funzionamento metacognitivo più agile, in grado di valutare rapidamente un più ampio numero di persone. La SVaM richiede infatti un notevole dispendio di tempo: nella formazione dei valutatori a utilizzare uno strumento concettualmente complesso; nella trascrizione fedele delle sedute audioregistrate; nelle valutazione stessa, effettuata su un numero elevato di sedute. Il gruppo ha sviluppato allora l'Intervista per la Valutazione della Metacognizione - IVaM (Semerari, d'Angerio, Popolo, Cucchi, Ronchi, Maffei, Dimaggio, Nicoló, \& Carcione, 2008), che consente la valutazione della metacognizione di un soggetto nel corso di un colloquio a sé stante, ripetibile semestralmente per successive valutazioni. L'IVaM è un'intervista semistrutturata, che ha lo scopo di sollecitare nell'intervistato e di valutare le sue abilità metacognitive; è composta da cinque parti: uno stimolo iniziale, e quattro serie di domande. Inizialmente si cerca di stimolare la produzione di una narrativa da parte dell'intervistato, racconto a partire dal quale vengono indagate le varie funzioni metacognitive; a tale scopo viene chiesto al paziente di 
descrivere l'episodio psicologicamente più difficile occorso negli ultimi sei mesi. Successivamente vengono poste delle domande per elicitare/valutare in successione le funzioni metacognitive del monitoraggio, differenziazione, integrazione e decentramento; non è stata considerata in questa prima versione dell'intervista l'area della mastery. Si tratta di domande predefinite, che in caso di difficoltà da parte dell'intervistato, possono essere ripetute o riformulate in modo da chiarirne il senso. Questo strumento presenta quindi delle caratteristiche che lo rendono più adeguato, ad esempio, a studi di esito in quanto permette lo studio di un più ampio numero di persone; si potrebbero così generalizzare le osservazioni ottenute sui diversi disturbi, ma anche approfondire lo studio sull'architettura della metacognizione in campioni significativi di pazienti e di soggetti non clinici.

\section{L'IVaM: gli studi principali}

L'IVaM è stata somministrata a 172 individui sani (Semerari, Cucchi, Dimaggio, Cavadini, Carcione, Bottelli, Siccardi, d'Angerio, Pedone, Ronchi, Maffei, \& Smeraldi, 2010) al fine di valutare, una volta raggiunta una buona inter-rater reliability, la validità di costrutto dello strumento elaborato a partire dalle quattro differenti funzioni metacognitive descritte. Dall'analisi fattoriale sono emersi due fattori: il primo include le capacità di mantenere una distanza critica dalle proprie convinzioni e di essere in grado di vedere il mondo dalla prospettiva dell'altro; il secondo fattore comprende invece le capacità del soggetto di riconoscere i propri stati interni, di integrarli in una rappresentazione coerente di sé e di distinguere differenti tipi di rappresentazioni (ad esempio fantasia e realtà). Questi risultati hanno delle implicazioni importanti sulla teoria della metacognizione. Nel complesso confermano infatti come le abilità che consentono di riflettere su di sé siano distinte da quelle che permettono di ragionare sugli altri. Il decentramento si aggrega infatti 
con alcuni aspetti della differenziazione, quelli relativi alla distanza critica, funzioni queste che richiedono al soggetto di assumere una posizione altra di osservazione. Nel secondo fattore troviamo invece le capacità di monitoraggio che, insieme a quelle che consentono di riconoscere la natura delle proprie rappresentazioni, si aggregano alle capacità di integrazione: questo in linea con l'idea che per avere una rappresentazione coerente di sé è necessario avere una corretta rappresentazione della propria esperienza. L'IVaM si è allora mostrata efficace nel sollecitare le funzioni metacognitive nei soggetti indagati e di distinguere tra processi di ragionamento relativi al sé e agli altri. Sono state allora studiate le proprietà di validità convergente dei due fattori emersi (Semerari, Dimaggio, Cucchi, Cavadini, d'Angerio, Battelli, Siccardi, Ronchi, Maffei, \& Smeraldi, 2010); il fattore che include il monitoraggio degli stati interni è stato correlato con il costrutto dell'alessitimia misurato dalla Toronto Alexithymia Scale (Bagby et al., 1994a, 1994b), il secondo invece con prove cognitive di teoria della mente. Nello stesso studio si è cercato di valutare, su un campione di 124 soggetti sani, se le funzioni metacognitive fossero correlate a tratti di personalità sottostanti; è stato valutato allora il narcisismo utilizzando il Narcissistic Personality Inventory (NPI; Raskin \& Hall, 1979; Raskin \& Terry, 1988). È emerso, coerentemente con le ipotesi cliniche (Dimaggio, Semerari, Falcone, Nicolò, Carcione, \& Procacci, 2002; Dimaggio, Semerari, Carcione, Nicolò, \& Procacci, 2007), che il monitoraggio correlava con tratti di narcisismo covert.

\section{Conclusioni}

Il III Centro è sorto ed è, in parte, rimasto un gruppo di clinici che inizialmente non avevano una formazione specifica nella ricerca e questo dato si rifletteva nei limiti metologici dei nostri lavori. L'impianto delle nostre ricerche era estremamente "time consuming" e adatto allo studio, sia pure particolareggiato, di pochi casi. Prese singolarmente, 
queste ricerche più che corroborare le ipotesi (theory-testing) potevano raffinarle (theory-building, si veda Stiles, 2006 per una distinzione tra i due tipi di ricerca) e valutarne la successiva estendibilità a campioni più ampi. Solo negli ultimi lavori l'esperienza acquisita col tempo e la collaborazione con ricercatori più esperti ci ha permesso di dimostrare la validità di costrutto e la validità convergente degli strumenti utilizzati, in particolare con ricerche effettuate nell'area della schizofrenia (Lysaker, Carcione, Dimaggio, Johannesen, Nicolò, Procacci, \& Semerari, 2005; Lysaker, Dimaggio, Buck, Carcione, \& Nicolò, 2007; Lysaker, Dimaggio, Buck, Carcione, Procacci, Davis, \& Nicolò 2010; Lysaker, Shea, Buck, Dimaggio, Nicolò, Procacci, Salvatore, \& Rand, 2010). Recenti lavori di analisi fattoriale applicata all'IVaM ci hanno permesso di iniziare a verificare le ipotesi formulate già negli anni '90 su quali fossero le diverse sottofunzioni metacognitive e sulle loro possibili correlazioni.

Se le consideriamo complessivamente, queste ricerche ci permettono oggi di trarre alcune precise conclusioni circa le ipotesi iniziali. Innanzitutto, riteniamo di aver portato prove sufficienti per sostenere la polifunzionalità della metacognizione. Mettiamo a confronto l'ipotesi olistica secondo cui la metacognizione è una grandezza unidimensionale descrivibile in termini di alta, media, bassa metacognizione con l'ipotesi polifunzionale, secondo cui è costituita da funzioni qualitativamente distinte; se anche in pochi casi, pazienti diversi sono danneggiati in funzioni diverse, questo rilievo dimostra che l'ipotesi monodimensionale è falsa. Ad esempio, possiamo avere un paziente abile nel descrivere $\mathrm{i}$ propri stati interni ma che non riesce ad assumere distanza critica dalle sue credenze e a riconoscerle come una rappresentazione del mondo che può essere falsa; un altro paziente può invece ammettere l'opinabilità delle sue credenze ma non essere in grado di descrivere le proprie rappresentazioni: la capacità di descrivere le rappresentazioni e 
la consapevolezza della loro natura soggettiva e opinabile non sono allora la stessa cosa.

Al tempo dei nostri primi lavori era difficile persino spiegare questo concetto. Oggi dati provenienti dalla social psychology e dalle neuroscienze convergono verso una descrizione della metacognizione nei termini di una funzione composta da abilità distinte (vedi Dimaggio \& Lysaker, 2010). Naturalmente, dimostrare che la metacognizione sia una grandezza composta da sottofunzioni distinte non significa dimostrare che queste siano esattamente quelle che abbiamo ipotizzato e che sono rappresentate nella struttura della SVaM e dell'IVaM. Da questo punto di vista, le analisi fattoriali applicate all'IVaM vanno nella direzione di un modello a due fattori. Il primo fattore può essere definito di Teoria della Mente (ToM) e include la tendenza a spiegare il comportamento attribuendo agli altri delle credenze e la consapevolezza che le credenze, comprese le proprie, sono rappresentazioni interne del mondo che possono essere false (ovvero non corrispondenti al mondo esterno). Tali caratteristiche confluiscono in un fattore dell'IVaM costituito dal decentramento (capacità di comprendere la mente altrui mettendosi nella prospettiva dell'altro) e dalla differenziazione (capacità di distinguere tra rappresentazione interna e mondo esterno). Il secondo fattore, che può essere definito dell'Autoriflessività, è costituito dal monitoraggio e dall'integrazione ovvero dalla capacità di riconoscere pensieri ed emozioni e dalla capacità di riflettere in modo integrato. Questi risultati correggono in parte alcune ipotesi iniziali sostenute nei lavori teorici del centro: quella che collocava la differenziazione all'interno dell'autoriflessività, e l'ipotesi di una marcata distinzione tra monitoraggio e integrazione. Un possibile modello a due fattori della metacognizione osservabile nella popolazione generale non esclude però la possibilità di considerare le sottofunzioni semi-indipendenti; anche le funzioni associate in un fattore possono avere un andamento diverso nella popolazione clinica. Se riprendiamo ad esempio i dati relativi alla 
correlazione tra basso monitoraggio, alessitimia e narcisismo covert (Semerari, Dimaggio et al., 2010), possiamo osservare come pazienti narcisisti covert tendano a evitare le relazioni, a differenza invece dei pazienti borderline che stabiliscono invece relazioni instabili e intense. Ipotizziamo allora che i fattori descrivono funzioni che operano in modo associato ma che possono disgiungersi in determinate situazioni cliniche.

Infine, se confermato da ulteriori ricerche, il modello a due fattori può avere importanti implicazioni terapeutiche. Ad esempio, l'associazione tra decentramento e differenziazione descrive quell'abilità metarappresentativa che consente di formarsi una rappresentazione delle proprie idee come visti da una terza persona: un osservatore "interno" nel caso della differenziazione ("penso questo ma se mi metto in un'altra prospettiva $\mathrm{mi}$ rendo conto che posso sbagliare"); un osservatore "esterno" nel caso del decentramento ("Mi mancano di attenzione, ma se esco dalla mia prospettiva e guardo il mondo con i loro occhi, mi rendo conto che sono pieni di problemi da affrontare e non potrebbero prestarmi l'attenzione che desidero"). Se si vuole aiutare un paziente ad assumere distanza critica da una propria credenza, non è necessario allora sollecitare operazioni autoriflessive per incrementare tale funzionamento. Può essere più utile formulare interventi che vadano a sollecitare in alcuni casi la differenziazione, in altri che incoraggino il paziente ad assumere la prospettiva degli altri, tutto questo in relazione al funzionamento specifico del paziente.

Resta da discutere la rilevanza clinica della distinzione delle sottofunzioni: disturbi in diverse funzioni metacognitive contribuiscono a creare diverse realtà cliniche e, se sì,, in che senso? Le ricerche condotte su soggetti schizofrenici da Lysaker, in parte anche con la nostra collaborazione, hanno evidenziato come la realtà clinica sia influenzata in modo diverso dallo specifico profilo metacognitivo del paziente. $\dot{E}$ stato infatti dimostrato che la metacognizione, correlata 
anche se parzialmente indipendente con la neurocognizione (Lysaker et al., 2005, 2007) e la funzione esecutiva (Lysaker, Warman, Dimaggio, Procacci, LaRocco, Clark, Dike, \& Nicolò, 2008), contribuisce a spiegare lo scarso funzionamento sociale dei pazienti (Lysaker, Shea et al., 2010). Inoltre è emerso nel corso di un trial clinico che avere capacità di assumere distanza critica (differenziazione) dalle proprie credenze è il crinale superato il quale si predice un migliore funzionamento in termini di outcome lavorativo (Lysaker et al., 2010). Tali dati di psicopatologia, uniti alla formalizzazione di modelli per promuovere progressivamente la metacognizione nei disturbi di personalità (Dimaggio et al., 2010; Dimaggio et al., in stampa), hanno portato nella schizofrenia alla recente proposta di modelli di trattamento che prevedono di promuovere prima le funzioni metarappresentative più basiche dove esse fossero carenti, e solo successivamente quelle a complessità crescente (Lysaker, Shea et al., 2010).

L'obiettivo negli anni futuri è aumentare ricerche cross-sectional nei disturbi di personalità, creare valori normativi che distinguano tra livelli metacognitivi adattivi e patologici e valutare in che modo la metacognizione sia compromessa in una serie di altri disturbi, quali disturbi post-traumatici, depressione o altri disturbi di asse I.

\section{Bibliografia}

Bagby, R.M., Parker, J.D.A., \& Taylor, G.J. (1994a). The twenty-item Toronto Alexithymia Scale-I. Item selection and cross-validation of the factor structure. Journal of Psychosomatic Research, 38, 23-32.

Bagby, R.M., Parker, J.D.A., \& Taylor, G.J. (1994b). The twenty-item Toronto Alexithymia Scale-II. Convergent, Discriminant, and Concurrent Validity. Journal of Psychosomatic Research, 38, 33-40.

Carcione, A., Dimaggio, G., Fiore, D., Nicolò, G., Procacci, M., Semerari, A., \& Pedone, R. (2008). An intensive case analysis of client metacognition in a good-outcome psychotherapy: Lisa's case. Psychotherapy Research, 18(6), 667-676. 
Ricerca in Psicoterapia / Research in Psychotherapy 2010; 2(13): 217-240 http://www.researchinpsychotherapy.net

Carcione, A., Falcone, M., Magnolfi, G., \& Manaresi, F. (1997). La funzione metacognitiva in psicoterapia: Scala di Valutazione della Metacognizione (SVaM) [Metacognitive function in psychotherapy: The Metacognition Assessment Scale (MAS)]. Psicoterapia, 9, 91-107.

Carcione, A., Semerari, A., Nicolò, G., Pedone, R., Popolo, R., Conti, L., Fiore, D., Procacci, M., \& Dimaggio, G. (2010). Metacognitive mastery dysfunctions in personality disorder psychotherapy. Psychiatry Research. Manuscript submitted for publication.

Cartwright-Hatton, S., \& Wells, A. (1997). Beliefs about worry and intrusions: the Meta-Cognitions Questionnaire and its correlates. Journal of Anxiety Disorders, 11, 279-296.

Clarkin, J.F., Yeomans, F.E., \& Kernberg, O.F. (Eds.). (1999). Psychotherapy for borderline personality. New York: Wiley.

Dimaggio, G., Carcione, A., Nicolò, G., Conti, L., Fiore, D., Pedone, R., Popolo, R., Procacci, M., \& Semerari, A. (2009). Impaired Decentration in Personality Disorder: A Series of Single Cases Analysed with the Metacognition Assessment Scale. Clinical Psychology and Psychotherapy, 16(5), 450-62.

Dimaggio, G., Carcione, A., Petrilli, M., Procacci, M., Semerari, A., \& Nicolò, G. (2005). States of mind organization in personality disorders. Typical states and the triggering of inter-state shifts. Clinical Psychology and Psychotherapy, 12, 346-359.

Dimaggio, G., Carcione, A., Salvatore, G., Semerari, A., \& Nicolò, G. (2010) A rational model for maximizing the effects of therapeutic relationship regulation in personality disorders with poor metacognition and overregulation of affects. Psychology and Psychotherapy: Theory, Research and Practice, 83(4), 363-384.

Dimaggio, G., \& Lysaker, P.H. (Eds.). (2010). Metacognition and severe adult mental disorders: From basic research to treatment. London: Routledge.

Dimaggio, G., Procacci, M., Nicolò, G., Popolo, R., Semerari, A., Carcione, A., \& Lysaker, P.H. (2007). Poor Metacognition in Narcissistic and Avoidant Personality Disorders: Four Psychotherapy Patients Analysed Using the Metacognition Assessment Scale. Clinical Psychology and Psychotherapy, 14, 386-401.

Dimaggio, G., Salvatore, G., Fiore, D., Carcione, A., Nicolò, G., \& Semerari, A. (in press). General principles for treating the overconstricted personality disorder. Toward operationalizing technique. Journal of Personality Disorders.

Dimaggio, G., \& Semerari, A. (2001). Psychopathological narrative forms. Journal of Constructivist Psychology, 14, 1-23.

Dimaggio, G., \& Semerari, A. (Eds.). (2003). I disturbi di personalità. Modelli e trattamento. Stati mentali, metarappresentazione, cicli interpersonali. RomaBari: Laterza.

Dimaggio, G., \& Semerari, A. (2004). Disorganized narratives: The psychological condition and its treatment. In L. Angus \& J. McLeod (Eds.), The handbook of narrative and psychotherapy: Practice, theory and research (pp. 263-282). Thousand Oaks, CA: Sage.

Dimaggio, G., Semerari, A., Carcione, A., Nicolò, G., \& Procacci, M. (2007). Psychotherapy of Personality Disorder: Metacognition, states of mind and interpersonal cycles. London: Routledge. 
Ricerca in Psicoterapia / Research in Psychotherapy 2010; 2(13): 217-240 http://www.researchinpsychotherapy.net

Dimaggio, G., Semerari, A., Falcone, M., Nicolò, G., Carcione, A., \& Procacci, M. (2002). Metacognition, states of mind, cognitive biases and interpersonal cycles. Proposal for an integrated model of Narcissism. Journal of Psychotherapy Integration, 12, 421-451.

Fonagy, P. (1991). Thinking about thinking: Some clinical and theoretical considerations in the treatment of a borderline patient. International Journal of Psycho-Analysis, 72, 639-656.

Fonagy, P., Gergely, G., Jurist, E.L., \& Target, M. (2002). Affect regulation, mentalization, and the development of the Self. New York: Other Press.

Fonagy, P., \& Target, M. (1996). Playing with reality: I. Theory of mind and the normal development of psychic reality. International Journal of Psychoanalysis, 77, 217-233.

Frith, C. (1992). The Cognitive Neuropsychology of Schizophrenia. Hillsdale, NJ: Erlbaum.

Guttman, H.A., \& Laporte, L. (2002). Empathy in families of women with borderline personality disorder, anorexia nervosa, and a control group. Family Process, 39, 345-358.

Honkalampi, K., Hintikka, J., Antikainen, R., Lehtonen, J., \& Viinamäki, H. (2001). Alexithymia in patients with major depressive disorder and comorbid cluster c personality disorders: A 6-month follow-up study. Journal of Personality Disorders, 3, 245-254.

Inoue, Y., Tonooka, Y., Yamada, K., \& Kanba S. (2004). Deficiency of theory of mind in patients with remitted mood disorder. Journal of Affective Disorders, 82, 403-409.

Inoue, Y., Yamada, K., \& Kanba, S. (2006). Deficit in theory of mind is a risk for relapse of major depression. Journal of Affective Disorder, 95, 125-127.

Kernberg, O.F. (1975). Borderline conditions and pathological narcissism. New York: Jason Aronson.

Krueger, R.F., Skodol, A.E., Livesley, J.W., Shrout, P.E., \& Huang, Y. (2007). Synthesizing dimensional and categorical approaches to personality disorders: Refining the research agenda for DSM-V axis II. International Journal of Methods in Psychiatric Research, 16, 65-73.

Liotti, G. (2002). The inner schema of borderline states and its correction during psychotherapy: A cognitive-evolutionary approach. Journal of Cognitive Psychotherapy, 16, 349-366.

Lysaker, P.H., Carcione, A., Dimaggio, G., Johannesen, J.K., Nicolò, G., Procacci, M., \& Semerari, A. (2005). Metacognition amidst narratives of self and illness in schizophrenia: Associations with insight, neurocognitive, symptom and function. Acta Psychiatrica Scandinavica, 112(1), 64-71.

Lysaker, P.H., Dimaggio, G., Buck, K.D., Carcione, A., \& Nicolò, G. (2007). Metacognition and the sense of self within narratives of schizophrenia: Associations with multiple domains of neurocognition. Schizophrenia Research, 93, 278-287.

Lysaker, P.H., Dimaggio, G., Buck, K.D., Carcione, A., Procacci, M., Davis, L.W., \& Nicolò, G. (2010). Metacognition and Schizophrenia: The capacity for self- reflectivity and prospective assessments of work performance over six months. Schizophrenia Research, 122, 124-130.

Lysaker, P.H., Shea, A.M., Buck, K.D., Dimaggio, G., Nicolò, G., Procacci, M., Salvatore, G., \& Rand, K.L. (2010). Metacognition as a mediator of the effects of impairments in neurocognition on social function in 
Ricerca in Psicoterapia / Research in Psychotherapy 2010; 2(13): 217-240 http://www.researchinpsychotherapy.net

schizophrenia spectrum disorders. Acta Psychiatrica Scandinavica, 122(5), 405-413.

Lysaker, P.H., Warman, D.M., Dimaggio, G., Procacci, M., LaRocco, V., Clark, L., Dike, C., \& Nicolò G. (2008). Metacognition in schizophrenia: Associations with multiple assessments of executive function. Journal of Nervous and Mental Disease, 196, 384-389.

Nicolò, G., Centenero, E., Nobile, S., \& Porcari, F. (2002) Disturbo paranoideo di personalità: ricerca su caso singolo. Ricerca in Psicoterapia, 5(1-2), 89107.

Perner, J., \& Wimmer, H. (1985). "John thinks that Mary thinks that...": attribution of second-order false-beliefs by 5 to 10-year-old children. Journal of Experimental Child Psychology, 5, 125-137.

Perris, C., \& Skagerlind, L. (1998). An integrated, multilevels, metacognitive approach to the treatment of patients with a schizophrenic disorder or a severe personality disorder. In C. Perris \& P.D. McGorry (Eds.), Cognitive psychotherapy of psychotic and personality disorders (pp. 197-211). Chichester: Wiley.

Raskin, R.N., \& Hall, C. S. (1979). A narcissistic personality inventory. Psychological Reports, 45(2), 590.

Raskin, R., \& Terry, H. (1988). A principal-components analysis of the narcissistic personality inventory and further evidence of its construct validity. Journal of Personality and Social Psychology, 54(5), 890-902.

Ryle, A. (1997). Cognitive analytic therapy and borderline personality disorder: The model and the method. Chichester: Wiley.

Ryle, A., \& Kerr, I.B. (2002). Introducing cognitive analytic therapy. Principles and Practice. Chichester: Wiley.

Semerari, A. (Ed.). (1999). Psicoterapia cognitiva del paziente grave. Metacognizione e relazione terapeutica. Milano: Raffaello Cortina Editore.

Semerari, A., Cucchi, M., Dimaggio, G., Cavadini, D., Carcione, A., Bottelli, V., Siccardi, T., D'Angerio, S., Pedone, R., Ronchi, P., Maffei, C., \& Smeraldi, E. (2010). The development of the Metacognition Assessment Interview: Instrument description, Factor Structure and Reliability in a non-clinical sample. Manuscript submitted for publication.

Semerari, A., Dimaggio, G., Cucchi, M., Cavadini, D., D’Angerio, S., Battelli, V., Siccardi, T., Ronchi, P., Maffei, C., \& Smeraldi, E. (2010). The Metacognition Assessment Interview: concurrent validity and association with attachment style and narcissism. Manuscript submitted for publication.

Semerari, A., Carcione, A., Dimaggio, G., Nicolo, G., Pedone, R., \& Procacci, M. (2005). Metarepresentative functions in borderline personality disorder. Journal of Personality Disorders, 19(6), 690-710.

Semerari, A., Carcione, A., Dimaggio, G., Falcone, M., Nicolò, G., Pontalti, I., \& Procacci, M. (2001). E possibile osservare diversi profili metacognitivi in diversi disturbi di personalità? Come misurare la funzione metacognitiva in psicoterapia. Sistemi Intelligenti, 12, 189-208.

Semerari, A., Carcione, A., Dimaggio, G., Nicolò, G., \& Procacci, M. (2007). Understanding minds: Different functions and different disorders? The contribution of psychotherapy research. Psychotherapy Research, 17, 106119.

Semerari, A, Carcione, A., Dimaggio, G., Falcone, M., Nicolò, G., Procacci, M., Alleva, G., \& Mergenthaler, E. (2003b). Assessing problematic states inside 
patient's narratives. The Grid of Problematic Conditions. Psychotherapy Research, 13(3), 337-353.

Semerari, A., Carcione, A., Dimaggio, G., Falcone, M., Nicolò, G., Procacci, M., \& Alleva, G. (2003). How to evaluate metacognitive functioning in psychotherapy? The Metacognition Assessment Scale and its applications. Clinical Psychology and Psychotherapy, 10, 238-261.

Semerari, A., d'Angerio, S., Popolo, R., Cucchi, M., Ronchi, P., Maffei, C., Dimaggio, G., Nicoló, G., \& Carcione, A. (2008). L'Intervista per la Valutazione della Metacognizione (IVaM): Descrizione dello Strumento. Cognitivismo Clinico, 5(2), 174-192.

Sperber, D. (2000). Metarepresentations. Oxford: Oxford Univeristy Press.

Stiles, W.B. (2006). Assimilation and the process of outcome: Introduction to a special section. Psychotherapy Research, 16, 389-392.

\title{
Metacognitive dysfunctions in personality disorders: a review of the principal researches of the Third Centre of Cognitive Psychotherapy in Rome
}

\begin{abstract}
Since mid 90's the Third Centre of Cognitive Psychotherapy in Rome has been interested in the research and treatment of severe and difficult to treat patients;. In this work we analyze some of the most important publications on this issue. These are researches on the therapeutic process led by the clinical observation which suggests that some specific metacognitive dysfunctions impede the recognition of own and others mental states; the regulation of the therapeutic alliance could enhance these dysfunctions making possible the interventions on the symptomatic aspects of the patient. There are sufficient proofs supporting that metacognition is composed of different half-independent subfunctions. Other studies of the group showed how the clinical reality of the serious patient is influenced by the presence of specific metacognitive dysfunctions.
\end{abstract}

\section{Keywords}

Metacognition, personality disorder, process research 